\title{
EMPYEMA THORACIS: Bacteriological analysis of pleural fluid from the largest chest hospital in Delhi
}

\author{
Jain Sonali ${ }^{1}$, Banavaliker J N ${ }^{2}$ \\ ${ }^{\prime}$ (Dept of Microbiology, RBIPMT, Delhi, India) \\ ${ }^{2}$ (Dept of Chest and TB, RBIPMT, Delhi, India)
}

\begin{abstract}
A retrospective microbiological review of pleural fluid samples was conducted to investigate the various bacteria associated with empyema thoracis and to monitor their antibiogram pattern in the 2 year study period. A total of 2219 pleural fluid samples were included in the study yielding 393 positive cultures, 302 pyogenic isolates and 107 tubercular isolates. An obvious stronghold of Gram negative bacteria in the pleural space was clearly demonstrated. A massive amount of antimicrobial resistance was noted among the pyogenic isolates increasing over time. A need for an appropriate antibiotic policy to herald judicious antibiotic use was strongly felt for.
\end{abstract}

Keywords- Antimicrobial resistance ,empyema, Gram negative bacteria, MBL, pleural fluid.

\section{Introduction}

The microbial etiology of pleural space infections has changed since the introduction of antibiotics [1]. Gram positive organisms are slowly and steadily losing their foothold in the pleural space to the more resilient and resistant gram negative bacteria (GNB) [2].

The discovery of antibiotics in the $20^{\text {th }}$ century marked a watershed in the treatment of infections. The spectacular success of antibiotics generated complacency, exemplified by the (now surprising) statement by William H Stewart US surgeon general that "it is time to close the book of infectious diseases" (Stewart 1967). However, this huge antibiotic created optimism has been misplaced. The microbial world has demonstrated remarkable resilience and have adapted to the antibiotic charged environment adopting the principle of "survival of the fittest" [3]. Local evolution of MDR (multi drug resistant) bacteria under pressure of excessive antibiotic use combined with horizontal gene transfer provides a means for rapid and widespread dissemination of resistant genomes like ESBLs (extended spectrum beta lactamases)[4]. The plasmids responsible for ESBL production frequently carry genes that encode for various resistance mechanisms and multiple ESBL enzymes that target various antibiotic classes, which dramatically reduce treatment options. Carbapenems, which are currently the treatment of choice for ESBLs, may thus be losing their effectiveness [5].

The present retrospective study was designed to review our experience with the microbial causes of empyema over a period of 02 years and their antibiotic resistance patterns. The knowledge of likely prevalent strains along with their antimicrobial resistance pattern helps in the framing of antibiotic policy and better management of patients.

\section{Aims And Objectives}

The aim of this study was to evaluate the various bacteria isolated from pleural fluids submitted to the microbiology laboratory at Rajan Babu Institute of Pulmonary Medicine and Tuberculosis (RBIPMT), Delhi. Further, the resistance patterns of the organisms isolated were also studied keeping in view the change in resistance trend, if any.

\section{Material And Methods}

All pleural fluid samples (from any cause) collected and transported without delay with universal safety precautions, to the microbiology laboratory for microbiological analysis were included in this study. Samples were obtained from both outpatient department and from patients admitted in our hospital from January 2010 to October 2011. Single or mixed growth from one patient and consecutive samples from new patients were included in this study. If repeat sample was received from a patient already enrolled, it was excluded.

All samples for pyogenic cultures were inoculated on 5\% sheep blood agar and Mac Conkey agar and a Gram stained smear from the sample was also made for presumptive diagnosis. The specimens were processed for isolation and identification based on standard laboratory techniques [6].

In case tubercular empyema was suspected, smear for Acid Fast Bacilli (AFB) was made and ZiehlNeelsen and fluorescent staining was done. The sample was inoculated on plain Lowenstein-Jensen medium . A slope of Lowenstein-Jensen medium with para-nitro benzoic acid was also inoculated and growth if any was identified based on standard laboratory techniques [6]. 
Antibiotic susceptibility testing of the pyogenic isolates was performed by Modified Kirby-Bauer disc diffusion technique using Mueller-Hinton agar.

3.1Detection of ESBL was done for GNB isolates by a preliminary screening and a confirmatory double disc synergy test (DDST) as recommended by Clinical laboratory standards institute (CLSI) [7].

3.2Detection of Methicillin resistant Staph aureus (MRSA) was done for Staphylococcal isolates using cefoxitin discs [7] and Macrolide-lincosamide -Streptogramin B inducible resistance (MLSB $\left.{ }_{i}\right)$ detection was done using clindamycin and erythromycin discs in the D test [8].

3.3Metallo- $\square$-lactamase (MBL) production was suspected when the isolate was resistant to carbapenems .Various methods have been recommended for screening MBL producers. These include the modified Hodge test, double disc synergy test using Imipenem and EDTA discs or ceftazidime and EDTA discs . For MIC detection the E strip test and the microdilution (microtitre) plate method is recommended [9]. We used the modified Hodge test in our study in view of its simplicity.

3.4Detection of AmpC - All the strains were screened for AmpC beta-lactamase production by the disc antagonism test. The isolates which showed reduced susceptibility to cefoxitin were tested for confirming AmpC production using the modified three-dimensional test. An indentation or flattening of the zone of inhibition indicated the AmpC production [10].

3.5MDR detection- Isolates were labeled as MDR if they were resistant to at least two classes of first line agents like ampicillin, trimethoprin-sulphamethoxazole, flouroquinolones, gentamicin, and cephalosporins (cefotaxime, ceftriaxone and ceftazidime) [11].

3.6Statistical analysis: For the retrospective analysis, SPSS software was used for calculation of percentages and WHONET ver5.5 was used for data analysis.

\section{Results}

A total of 2219 pleural fluid samples were received in the microbiology laboratory from Jan2010 to Oct2011 for culture and sensitivity. Out of the 2219 samples, 393 samples (17.7\%) yielded a positive specimen. Almost all of the pleural space infections were pyogenic in origin $(n=286,72.7 \%$ of the total positive cultures) producing a total of 302 isolates. A tubercular origin of infection was noted in 107(27.2\%) samples.

Of the 302 pyogenic isolates, Gram negative bacteria were in the forefront with 269 organisms $(89.02 \%)$ and Gram positive bacteria contributed a meager $9.9 \%(n=30)$. Thus, a clear cut dominance of GNB in the pleural space was noted in our retrospective review. The various organisms isolated are shown in "Table-1"

TABLE 1- Spectrum of various organisms isolated

\begin{tabular}{|l|l|l|}
\hline Organism & No. Of isolates & \% of isolates \\
\hline $\begin{array}{l}\text { Pseudomonas } \\
\text { aeruginosa }\end{array}$ & 167 & $55.2 \%$ \\
\hline Enterobacteriaceae & 101 & $33.4 \%$ \\
\hline Klebsiella spp & 66 & $21.8 \%$ \\
\hline E.coli & 20 & $6.6 \%$ \\
\hline Enterobacter cloacae & 09 & $3.9 \%$ \\
\hline Proteus mirabilis & 06 & $1.9 \%$ \\
\hline Staphylococcus aureus & 29 & $9.6 \%$ \\
\hline $\begin{array}{l}\text { Others(Acinetobacter } \\
\text { baumanni,Streptococcus } \\
\text { pneumonia,Candida } \\
\text { spp) }\end{array}$ & 05 & $1.6 \%$ \\
\hline M.tuberculosis & & $27.2 \%$ \\
\hline
\end{tabular}

The total prevalence of various beta-lactamases in our study amongst the 269 GNB isolated was found to be $41.6 \%$. A major chunk of this alarming resistance was contributed by the Enterobacteriaceae group (63.3\%) and the rest by the nonfermenters ( $28.7 \%$ ). Amongst the beta-lactamases identified, ESBL production was found to be the maximum (41.8\%) followed by MBL (9.6\%) producers and then the AmpC producers $(7.9 \%)$. The prevalence of the various beta-lactamases is shown in "Table-2" 
TABLE 2- Prevalence of various beta-lactamases

\begin{tabular}{|l|l|l|}
\hline Beta-lactamase & Organism producing & \% of isolates \\
\hline ESBL & E.coli & $50 \%$ \\
\hline & Klebsiella spp & $48.4 \%$ \\
\hline MBL & E.coli & $20 \%$ \\
\hline & Klebsiella spp & $15 \%$ \\
\hline & Pseudomonas aeruginosa & $07 \%$ \\
\hline AmpC & E.coli & $10 \%$ \\
\hline & Klebsiella spp & $9 \%$ \\
\hline MRSA & Staphylococcus aureus & $79.3 \%$ \\
\hline MLSBi & Staphylococcus aureus & $3.4 \%$ \\
\hline
\end{tabular}

Out of the 167 Pseudomonas aeruginosa isolates, 48(28.7\%) showed resistance to imipenem. The noteworthy and disturbing observation was the almost trebling of the carbapenem resistance observed in this group from $2010(n=12)$ to $2011(n=36)$. Of these 48 carbapenem resistant strains tested for MBL production, $12(7 \%)$ were found to be MBL positive (03 strains isolated in 2010 \& 09 strains in 2011). The remaining 36 carbapenem resistant strains that were MBL negative could be harboring alternate mechanisms of carbapenem resistance, like drug efflux pumps, low degree of outer membrane permeability and loss of OprD. No ESBL producers were seen in Pseudomonas group.

MDR organisms were also isolated with Enterobacteriaceae group contributing 35\% isolates (E.coli $20 \%$ and Klebsiella spp 15\%) followed by Pseudomonas aeruginosa ( $14.3 \%$ ). Of the 10 ESBL producing E.coli isolated, 4 were MDR ( 40\%). MBL production was seen in 4 isolates (20\% of E.coli isolated). 10 of the ESBL producers in Klebsiella spp were MDR (31.25\%) and 10 Klebsiella spp isolates were MBL producers. 24 of the 167 Pseudomonas aeruginosa isolates were MDR.

Polymicrobial infection was noted in 16 patients (4\% of the positive cultures) and was mostly due to the synergy of Klebsiella spp and Pseudomonas aeruginosa. Most of the E.coli and Staphylococcus aureus strains isolated were in pure cultures.

\section{Discussion}

Empyema is a well recognized clinical entity as early as the era of Hippocrates [12]. Despite the impact antibiotics have had on empyema, it still remains a common illness with significant morbidity and mortality. The emergence of antibiotic- resistant organisms , the increase in the frequency of nosocomial infections, and the steadily increasing number of patients with a compromised immunity have combined to keep pleural infections a common entity [13].

The reported spectrum of microorganisms responsible for empyema is varied, and is modified by introduction of antibiotics, patient specific factors such as surgical procedures, trauma or underlying conditions or by methodological factors namely the proper specimen collection, transport and culture. For these reasons, several studies have found discordant results in the spectrum of pathogens causing pleural space infections [1].

In the present retrospective review conducted at one of Asia's largest Chest and Tuberculosis hospital (1155 bedded), comprising 2219 pleural fluid samples received in the microbiology laboratory the percentage of positive cultures was $17.7 \%$. Rates of microbiological diagnosis in earlier studies have shown a wide variation. A lower positive culture rates similar to ours has been observed in Indian studies like that of Mohanty et $\mathrm{al}(15.3 \%)$ [14] and western studies like Ferrer et al(15.5\%) [15], Walshe et al(3.5\%) [16] and Barnes et al(1.4\%) [16].A high turnover rate of pleural fluid cultures varying from 31-89\% have been reported by various workers across the world [2,13,14,17-25]. A retrospective review done at Mayo medical centre, Rochester on the low yield of microbiological studies on pleural fluid specimens by Barnes et al including 1320 samples had a meagre $19(1.4 \%)$ true positive results. The reason for this wide disparity in positivity rates of empyema fluids were attributed to differences in techniques, antibiotic use, or the prevalence of effusions caused by infective processes. Some of the variations are likely explained by the differences in study population [16]. These may hold true in our setting also.

In our series, pleural fluid was found to be sterile in $82.2 \%$ cases. Here again, a wide variation in negative cultures has been observed worldwide ranging from as low as 7.3\% [13] to 98.5\% [16]. A low yield of pleural fluid cultures like ours is in concurrence with the studies by Delikaris et al (82.9\%) [12] and Mohanty et al $(84.2 \%)$ [14]. Given the low positive yield of pleural fluid cultures, we tried to determine the factors associated with a higher likelihood of positive results. Microbiological studies performed only on exudative effusions could definitely enhance the yield of the samples. This however, is not a practical solution since the exudative nature of the pleural fluid can't be ascertained prior to the fluid result being available. Second important factor in the low yield of pleural fluid samples could be the empiric administration of antibiotics to the patients before thoracocentesis, likely decreasing the yield of the cultures[16]. 
The paucity (absence) of anaerobes in our series is notable and could be an incriminating factor in the low culture positivity rate. The incidence of anaerobic isolates depends both on the care with which they are searched for and on the type of population studied (e.g. aspiration prone patients) . Inadequate methods to collect and transport the pleural specimens to the laboratory and technical difficulty of growing anaerobes could also have contributed to our missing exclusively anaerobic pathogens [1,12].

Our study highlights the emergence of aerobic gram negative bacteria as the predominant pathogens in empyema. Out of the 302 pyogenic isolates, 267(88.4\%) were aerobic GNB. A similar high rate of isolation of GNB from pleural fluid cultures was reported in India by Gupta S K et al(84.6\%) [26], Mohanty et al(86.4\%) [14], Goel et al (95.6\%) [20], Gagneja et al (91.07\%) [21] and Acharya et al (27.5\%) [23]. Prior to the availability of antibiotics, S. pneumoniae and $S$. pyogens accounted for most of the empyema cases[23]. After the discovery and widespread use of antibiotics in the 1940s, Staph aureus succeeded S. pneumoniae and S. pyogens as the major cause of empyema. Since the advent of beta-lactamase resistant semi-synthetic penicillins in the 1960s, the incidence of staphylococcal empyema has decreased and infections due to aerobic GNB (E.coli, Klebsiella spp, Pseudomonas spp, and Proteus spp) and anaerobes have increased markedly. These observations are in consensus with the reports of various workers who have emphasized the emergence of GNB as the predominant pathogen $[2,14,19,20,23-, 25,27]$. The isolation of aerobic GNB or multiple pathogens from pleural fluid is associated with a poor prognosis and indicates a more aggressive antimicrobial chemotherapy in contrast to the empyema caused by Gram positive pathogens[2].

More than one isolate was seen in 16 patients (4\%) mostly due to a combination of isolates from the Enterobacteriaceae group and Pseudomonas aeruginosa. A polymicrobial etiolgy of empyema thoracis is also well documented [18]. A similar combination was also reported by Dorobat OM et al [19]. A polymicrobial etiology of empyema has been documented to be varying from as low as 7.5\%[23] and 8] [21[ in Indian settings to up to $40.4 \%$ in the west $[11,16-18,20,24]$.

The most frequent isolate in our study population was Pseudomonas aeruginosa $(\mathrm{n}=167,55.3 \%$ of the total pyogenic isolates) a finding in agreement with many Indian [20,21] and western reports [19]. Gram negative enteric bacteria (33.4\%) were the next most common; the once predominant Gram positive organisms were conspicuous by their absence. This is comparable with earlier reports where streptococcus was rarely if ever seen as a cause of empyema[18].

Tubercular etiology was found in 107 patients (27.2\% of the total 393 positive). Gupta and coworkers [26] have reported the incidence of tubercular empyema to be $29 \%$ in 1989. A few studies from India like Banga et al [18] do report a high incidence of tubercular empyema akin to the figures from the west where isolation rates of $M$. tuberculosis from pus has been very high. Indian studies by and large have consistently reported a low isolation rate. Jha and co-workers (1979) found AFB in empyema in only 01 out of the 24 patients $(4.2 \%)$, whereas in a relatively recent series AFB could not be isolated from any of the 29 patients with empyema [18]. A similar total absence of M.tuberculosis was noted in a large scale study involving 1320 pleural fluid samples in the west by Barnes et al [16]. The cause of this phenomenon is not clear and necessitates further evaluation .

MRSA was reported at the rate of $79.3 \%$ in our study. Reported prevalence from different parts of the country varies from $30-85 \%$ and so far vancomycin resistance has not been reported barring a single report [28].

In our study the prevalence of various beta-lactamases in the GNB was found to be $41.6 \%$ which was alarmingly high. The ESBL (41.8\%) production was found to be greatest as compared to other beta-lactamases. Previous studies from India have reported have reported ESBL production to be between 6.6-68\% [28, 29]. ESBL and AmpC production was maximum in E.coli followed by Klebsiella spp as observed in various reports worldwide [29]. This increased number of AmpC producers is in agreement with other works from India which reported $17.3 \% \mathrm{AmpC}$ producers in Kolkata [10] and 22.9\% by Bandekar et al [10]. This rising ESBL production poses a daunting challenge in gram negative antibiotic resistance, indicative of an ominous trend of more and more isolates acquiring the resistance mechanisms. The antimicrobial armamentarium is thus, rendered ineffective.

A distressing situation arose when we analysed our data for carbapenem resistance among the Pseudomonas aeruginosa isolates. There was almost a threefold increase in carbapenem resistance in the 02 year study period. In numerous studies across the world, varying resistance (4-60\%) towards carbapenems has been documented $[9,30]$. A prevalence of carbapenem resistance from $31-64 \%$ has been reported from India too $[19,28]$. The possible reason for the manifold increase in carbapenem resistance in our institute may be the selective pressure created by the injudicious use of carbapenems in the wake of increasing ESBLs [19].

ESBL hydrolyse beta-lactam antibiotics. The plasmids responsible for ESBL production frequently carry genes that encode for various resistance mechanisms and multiple ESBL enzymes that target various antibiotic classes including carbapenems which dramatically reduces antibiotic options [5]. Resistance to carbapenems can be mediated by many mechanisms including decreased membrane permeability and increased efflux. However, production of metallo- $\beta$-lacatamase has assumed significance in the recent years. MBLs are 
often produced in combination with serine beta-lactamases such as pencillinase and cephalosporinase and as long as bacteria can survive with serine beta-lactamases at their disposal, production of MBL is not required [31]. The rampant and injudicious use of this last resort group of antibiotics by virtue of selection pressure has created a niche for the MBL producers and the MDR organisms. Studies have shown that a shift in the empirical therapy to carbapenems, due to the presence of ESBL producers, is associated with emerging resistance in Pseudomonas spp and the ESBL producing organisms themselves. This problem compounded by the horizontal transfer of genetic material between various Gram negative bacteria , increasing mobility of modern society, demographic changes, deteriorating hygiene and institutional opportunities e.g. day care centres, old age homes, hospices etc. ensures a steady supply of resistant clones of human pathogens [5]. Carbapenems are frequently used as the last choice drug in treating serious infections caused by GNB. Their failure combined by the diminishing number of new antibiotics in the pharmaceutical pipeline makes this observation a point of acute concern.

In the index study, of the 167 Pseudomonas isolates 12 were MBL producers (7\%). The prevalence of MBL in the present study is in concordance with the published literature [10, 32]. Amongst the imipenem resistant Pseudomonas isolates 25\% were MBL producers while mechanisms other than MBL production operated in the remaining 75\% imipenem resistant isolates. Navneeth et al from India, first reported MBL production in Pseudomonas aeruginosa to be 12\% in 2002 [9]. Since then the incidence of MBL producers has been reported to range from 7-65\% from various clinical specimens across the country. In the study conducted by Varaiya et al, 20.8\% of Pseudomonas aeruginosa isolates were MBL producers from various clinical specimens of which, a majority (30\%) belonged to the respiratory tract [9]. Another study conducted by Shashikala et al reported 20.7\% carbapenem resistant Pseudomoas isolates from endotracheal aspirates showing indwelling devices as a major risk factor for development of resistance in Pseudomonas spp [9]. Pseudomonas aeruginosa possess the ability to form biofilms that increases its virulence. Bacteria within biofilms are often less susceptible to antibiotics thus, antibiotics may select them enhancing their virulence. It is likely that most infections associated with foreign bodies (like ventilator associated pneumonia, catheter associated infections, chest tubes etc.) involve biofilms. Besides MBL production, this may the one of the other factor contributing to the resistance in Pseudomonas spp [5].

Among the bacterial isolates, highest percentage of MDR belonged to the Enterobacteriaceae (35\%) followed by Pseudomonas aeruginosa (14.3\%).

Thus, clearly more resilient and dangerous Gram negative pathogens have established themselves in nosocomial settings. Since most organisms that are carbapenem resistant are enteric bacteria, the obvious route of infection would be the faeco-oral route. Therefore, in hospitals patients who are harbouring MBL positive organisms should be kept isolated. It is of paramount importance to use antibiotics judiciously, collect data of local resistance patterns, perform prescription auditing and follow good infection control practices as the pipeline for newer antibiotics is fast drying up.

Lastly we would like to state the limitations of our study. Firstly, being a retrospective study it was prone to missing data. Secondly, since we lacked a molecular set up in our institute, we were unable to validate our findings genotypically. Thirdly, since detection methods for MBL and AmpC mediated resistance have not been standardised by CLSI, there may be discrepant results.

\section{Conclusion}

In the battle between bacteria and mankind, bacteria are constantly evolving newer mechanisms of resistance which makes the latest group of antibiotics ineffective. The strategy to win this battle has to be multipronged:

- Surveillance of antibiotic use and resistance rates

- Instituting appropriate antimicrobial stewardship programmes (ASP)

- The role of direct susceptibility testing in rapid initiation of appropriate antimicrobial therapy needs evaluation

- Promotion of health care practices in health institutions

- Application of logic in antibiotic selection for critically ill patients rather than a "tradition-based" approach for selecting treatment

- Lastly, the realization that antibiotics may not be the only answer for antimicrobial resistance

The need of the hour is for the medical community to accept this as "A time for challenge and opportunity".

Dr Jyoti Iravane, Dr A S Damle for being my mentors.

\section{Acknowledgements}

Dr Ravish Gupta and Mr John Thomas, without whose support this work would not be possible 


\section{References}

[1] J.M. Porcel, P. Vázquez, M. Vives, A. Nogués, M. Falguera, A. Manonelles: Pleural Space Infections: Microbiologic and Fluid Characteristics in 84 Patients. The Internet Journal of Pulmonary Medicine. 2003 Volume 3 Number 1.

[2] Chen KY, Hsueh PR, Liaw YS, Yang PC, Luh KT.A 10-year experience with bacteriology of acute thoracic empyema: emphasis on Klebsiella pneumoniae in patients with diabetes mellitus. Chest. 2000 Jun;117(6):1685-9.

[3] D Raghunath.Emerging antibiotic resistance in bacteria with special reference to India: J.Biosc.33(4),November 2008,593-603

[4] Peter M. Hawkey, Annie M Jones, The changing epidemiology of resistance. Journal of antimicrobial chemotherapy (2009) 64, suppl 1, i3-i10

[5] Robert E Siegel, Emerging Gram-Negative Antibiotic Resistance: Daunting Challenges, Declining Sensitivities, and Dire Consequences; Respiratory Care, April 2008 VOL 53 NO 4;471-9

[6] Betty A Forbes, Daniel F.Sahm, Alice S Weissfeld, bailey \& scott's diagnostic microbiology, 12 e (Mosby,2007)

[7] M100-S23, Performance Standards for Antimicrobial Susceptibility Testing; Twenty-Third Informational Supplement, CLSI

[8] Fiebelkorn KR, Crawford SA, McElmeel ML, Jorgensen JH. Practical disk diffusion method for detection of inducible clindamycin resistance in Staphylococcus aureus and coagulase-negative staphylococci. J Clin Microbiol. 2003;41:4740-4

[9] Ami Varaiya, Nikhil Kulkarni, Manasi Kulkarni, Pallavi Bhalekar \& Jyotsana Dogra , Incidence of metallo beta lactamase producing Pseudomonas aeruginosa in ICU patients: Indian J Med Res 127, April 2008, pp 398-402

[10] Loveena Oberoi, Nachhatarjit singh, PoonamSharma, Aruna Aggarwal, ESBL, MBL and Ampc $\beta$ Lactamases Producing Superbugs - Havoc in the Intensive Care Units of Punjab India: JCDR/2012/5016:2497

[11] Tsang KY, Leung WS, Chan VL, Lin AW, Chu CM ,Complicated parapneumonic effusion and empyema thoracis: microbiology and predictors of adverse outcomes. Hong Kong Med J. 2007 Jun;13(3):178-86

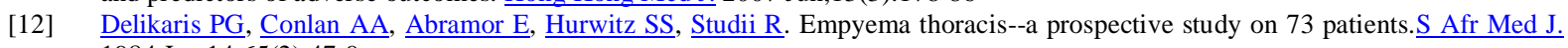
1984 Jan 14;65(2):47-9.

[13] Alfageme I, Muñoz F, Peña N, Umbría S. Empyema of the thorax in adults. Etiology, microbiologic findings, and management: Chest. 1993 Mar;103(3):839-43

[14] Srujana Mohanty, Arti Kapil, Bimal K Das.Bacteriology of parapneumonic pleural effusions in an Indian hospital: Trop Doct 1 October 2007 vol. 37(4),228-229

[15] Ferrer A, Osset J, Alegre J, Suriñach JM, Crespo E, Fernández de Sevilla T et al, Prospective clinical and microbiological study of pleural effusions. Eur J Clin Microbiol Infect Dis. 1999 Apr;18(4):237-41

[16] Barnes TW, Olson EJ, Morgenthaler TI, Edson RS, Decker PA, Ryu JH. Low yield of microbiologic studies on pleural fluid specimens. Chest. 2005 Mar;127(3):916-21.

[17] Julian A. Smith, Michael H. Mullerworth, ,George W. Westlake, and James Tatoulis. Empyema Thoracis: 14-Year Experience in a Teaching Center: Ann Thorac Surg 1991;51:39-42

[18] Amit Banga, GC Khilnani, SK Sharma, AB Dey, Naveet Wig and Namrata Banga, A study of empyema thoracis and role of intrapleural streptokinase in its management: BMC Infectious Diseases 2004, 4:19

[19] Dorobăt OM, Moisoiu A, Tălăpan D, Bacteria isolated from pleural fluid and their resistance to antimicrobials: Pneumologia. 2006 Apr

[20] 20.Goel N, Chaudhary U, Aggarwal R, Bala K. Antibiotic sensitivity pattern of gram negative bacilli isolated from the lower respiratory tract of ventilated patients in the intensive care unit. Indian J Crit Care Med 2009;13:148-51-Jun;55(2):47-51

[21] Deep Gagneja, Nidhi Goel, Ritu Aggarwal, and Uma Chaudhary Changing trend of antimicrobial resistance among gram-negative bacilli isolated from lower respiratory tract of ICU patients: A 5-year study,Indian J Crit Care Med. 2011 Jul-Sep; 15 (3): $164-167$.

[22] Dass R,Deka NM,Barman H, Duwarah SG,Khyriem AB, Sailia M K, Empyema thoracis: analysis of 150 cases from a tertiary care centre in north East India: Indian J Paediatr 2011Nov;78(11):131-7

[23] Preetam Rajgopal Acharya and Kusum V. Shah, Empyema thoracis: A clinical study;Ann Thorac Med. 2007 Jan-Mar; 2(1): 14-17.

[24] Kelly JW, Morris MJ, Empyema thoracis: medical aspects of evaluation and treatment. South Med J. 1994 Nov;87(11):1103-10

[25] Lin YC, Tu CY, Chen W, Tsai YL, Chen HJ, Hsu WH et al, An urgent problem of aerobic gram-negative pathogen infection in complicated parapneumonic effusions or empyemas; Intern Med. 2007;46(15):1173-8.

[26] Gupta S K, Kishan J,Singh S P, Review of one hundred cases of Empyema thoracis; Indian J Chest Dis Allied Sci; 1989 JanMar;31(1):15-20

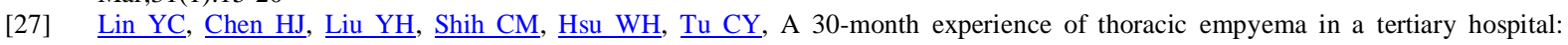
emphasis on differing bacteriology and outcome between the medical intensive care unit (MICU) and medical ward; South Med J. 2008 May;101(5):484-9.

[28] . Mishra A.P, You +1 'd this publicly. Undo

[29] Beta-Lactamase Threat in Respiratory Tract Infections: Focus onCephalosporin- Clavulinic Acid. Medicine update 2012 availableat www.apiindia.org/pdf/medicine_update.../infectious_disease_02.pdf. Acessed on 29th Sept 2012.

[30] Shiju M P, Yashavanth R, Narendra N .Detection of Extended spectrum betalactamase production and multidrug resistance in clinical isolates of E.coli and K.pneumoniae in Mangalore. Journal of Clinical and Diagnostic Research [serial online] 2010 June [cited: 2010 June 12]; 4: 2442 - 2445.

[31] Dorobăț OM, Moisoiu A, Tălăpan D, Incidence and resistance patterns of pathogens from lower respiratory tract infections (LRTI); Pneumologia. 2007 Jan-Mar;56(1):7-15.

[32] 31.Tariq Butt, Muhammad Usman, Rifat Nadeem Ahmad, Imran Saif. Emergence of Mettalo-beta-lactamse producing Pseudomonas aeruginosa in Pakistan, J Pak Med Assoc ,2005;Vol 55(7):301-4

[33] 32. Prashant Durwas Peshattiwar and Basavaraj Virupaksappa Peerapur, ESBL and MBL Mediated Resistance in Pseudomonas Aeruginosa: Journal of Clinical and Diagnostic Research. 2011 December, Vol-5(8): 1552-1554 